\title{
Evidenzbasierte Praxis als Grundlage sprachtherapeutischer Entscheidungen
}

\author{
Evidence-Based Practice as a Tool for Decisions in Speech- \\ Language Therapy
}

Holger Grötzbach

\begin{abstract}
Während Clinical Reasoning (CR) das Ziel hat, therapeutische Denk- und Entscheidungsprozesse zu untersuchen, ist es Aufgabe der evidenzbasierten Praxis (EBP), Forschungsergebnisse in den sprachtherapeutischen Alltag zu übertragen. Zwischen CR und EBP besteht eine enge Beziehung. So stellt die EBP Effektivitätsnachweise und medizinische Leitlinien zur Verfügung, um Sprachtherapeuten bei der Wahl wirksamer Behandlungsmethoden zu unterstützen. Sie stärkt außerdem Therapeuten darin, ihre Expertise bei der Lösung klinischer Probleme zu nutzen und auf die Präferenzen ihrer Patienten zu achten.
\end{abstract}

\section{LERNZIEL}

Der Beitrag vermittelt zum einen die Bedeutung, die der Effektivität in der Sprachtherapie zukommt. Zum anderen stellt er diejenigen Quellen vor, die für die Planung effektiver Therapien genutzt werden können. Schließlich wird auf einige Therapieprinzipien eingegangen, die sich für die Behandlung von Aphasien als effektiv erwiesen haben.

\section{Effektivität in der Sprachtherapie}

Effizienz und Effektivität. Nach Beushausen [1] untersucht das Clinical Reasoning (CR) die Denk-, Handlungs- und Entscheidungsprozesse, mit denen medizinische Professionelle v. a. das Ziel verfolgen, effiziente und effektive Behandlungen durchzuführen. Dabei gibt die Effizienz als Maß die Wirtschaftlichkeit einer Behandlung an: Ideal ist es, wenn mit vorgegebenen Ressourcen ein maximaler (medizinisch-therapeutischer) Nutzen erreicht wird. Im Unterschied dazu spiegelt die Effektivität die Wirksamkeit einer Therapie wider. Sie wird ermittelt, indem das Therapieergebnis mit dem jeweiligen Therapieziel verglichen wird. Je mehr das Ergebnis mit dem Ziel übereinstimmt, desto effektiver ist die Therapie. Den Therapiezielen kommt somit eine entscheidende Bedeutung zu. Sie müssen, um evaluiert werden zu können, genau, d. h. messbar, sein. Bleiben sie vage, sind eine Evaluation und damit ein Wirksamkeitsnachweis nicht möglich [2].

\footnotetext{
Merke

Die Effektivität einer Therapiemaßnahme lässt sich nur dann nachweisen, wenn das Therapieziel messbar definiert wird.
}

Effektivität versus Effizienz Wenn Effektivität und Effizienz miteinander in Beziehung gesetzt werden, dann ergibt sich, dass die Effektivität wichtiger ist als die Effizienz. Denn die Wahl einer unwirksamen Therapie stellt selbst dann eine verschwendete Ressource dar, wenn sie kostengünstig durchgeführt wird. Das CR muss sich daher v.a. darauf konzentrieren, die Effektivität einer Therapie sicherzustellen. Damit wird zum einen den Erwartungen der Patienten entsprochen, die sich von der Sprachtherapie eine (schnelle) Beseitigung oder zumindest eine (rasche) Reduktion ihrer jeweiligen Störung erhoffen [3]. Zum anderen helfen Effektivitätsnachweise, wenn eine Entscheidung zu legitimieren ist oder wenn Unsicherheiten bei einer Entscheidung vorliegen. So kann sich die Sprachtherapeutin in dem folgenden Beispiel auf Effektivitätsdaten berufen und dadurch ihre Argumentation untermauern: 
FALLBEISPIEL 1: EFFEKTIVITÄT VON APHASIETHERAPIE

Die Sprachtherapeutin Frau A. wird von der Ehefrau eines Patienten kontaktiert, der seit mehreren Jahren als Folge eines Schlaganfalls unter einer ausgeprägten Aphasie leidet. Die Ehefrau berichtet, dass ihr Mann schon seit längerer Zeit keine Sprachtherapie mehr erhalten habe. Sie möchte jetzt jedoch einen neuen Therapieversuch starten, um ihrem Mann die Chance zu geben, weitere sprachliche Fortschritte zu erreichen.

Frau A. wendet sich daraufhin an den behandelnden Hausarzt des Patienten mit der Bitte, Sprachtherapie zu verordnen. Der Hausarzt lehnt die Bitte zunächst mit dem Argument ab, dass es nach den vielen Jahren der Erkrankung mehr als unwahrscheinlich sei, Verbesserungen zu erzielen. Frau A. verweist jedoch auf den Inhalt der Aphasie-Leitlinie [4] und auf neue Studienergebnisse [5, 6], nach denen mit einer hochfrequent durchgeführten Aphasietherapie auch dann noch Fortschritte erreicht werden, wenn die Sprachstörung schon seit Jahren besteht. Der Hausarzt akzeptiert den Hinweis und sagt zu, eine Verordnung gemäß den Empfehlungen der Aphasie-Leitlinie auszustellen.

\section{Merke}

Der Effektivität kommt im Vergleich zur Effizienz in der Evaluation sprachtherapeutischer Maßnahmen eine höhere Priorität zu.

\section{Planung effektiver Therapien}

Wissensquellen Bei der Planung einer effektiven Therapie können Sprachtherapeuten auf das Wissen zurückgreifen, das sie in ihrer Ausbildung sowie in Fortund Weiterbildungen erworben haben. Außerdem stehen ihnen (wissenschaftlich ermittelte) Evidenzen zur Effektivität von Therapiemethoden zur Verfügung, die in Fachzeitschriften, Büchern und medizinischen Leitlinien veröffentlicht werden. Schließlich können sie noch Kollegen um Rat fragen und auf ihre Expertise bauen. Trotz der verschiedenen Wissensquellen bleibt jedoch die Herausforderung, sich für ein bestimmtes therapeutisches Vorgehen zu entscheiden. Denn für die Behandlung einer Störung gibt es in der Regel nicht nur eine, sondern mehrere therapeutische Optionen, die nicht selten in Konkurrenz zueinanderstehen. Da sich leicht vorhersagen lässt, dass für die Mehrheit der konkurrierenden Methoden keine zuverlässigen Evidenznachweise zur Verfügung stehen [7], wird die Wahl einer Therapieoption in der Praxis weniger auf Effektivitätsnachweisen und mehr auf der Erfahrung der Sprachtherapeuten beruhen.

\section{Merke}

Für die Planung einer effektiven Therapie stehen verschiedene Wissensquellen zur Verfügung. Sie umfassen die eigene Expertise, wissenschaftlich ermittelte Evidenzen und Leitlinien.

Patientenpräferenzen Obwohl die Effektivität bei der Bestimmung einer Therapiemethode zweifelsohne eine wichtige Rolle spielt, kann sie nicht das einzige Entscheidungskriterium sein. Der Grund dafür liegt darin, dass die Patienten mit einer gewählten Methode einverstanden sein müssen. So macht es bspw. keinen Sinn, einem Patienten verdickte Getränke zu verordnen, wenn der Patient das Verdickungsmittel ablehnt. Ebenso sinnlos ist es, eine hochfrequente Aphasietherapie zu planen, wenn ein Patient dazu nicht bereit ist. Mehr noch: Wie das folgende Beispiel zeigt, kommt dem Willen der Patienten eine höhere Bedeutung zu als jeder noch so gut begründeten therapeutischen Entscheidung.

\section{Merke}

Die Präferenzen eines Patienten haben Vorrang vor jeder auch noch so gut begründeten therapeutischen Entscheidung.

Sprachtherapeutische Expertise Die therapeutischen Optionen werden jedoch nicht nur durch den Willen der Patienten, sondern auch durch ihr Leistungsniveau beeinflusst. So kann es sein, dass die Aufmerksamkeitsund Gedächtniskapazitäten eines Patienten nicht ausreichen, um das Mendelsohn-Manöver zu erlernen. Umgekehrt kann der Fall auftreten, dass ein Patient eine Behandlung als kindisch empfindet, da das Therapieniveau weit unterhalb seiner Leistungsgrenze liegt. Es gehört zur Expertise der Sprachtherapeuten, das Leistungsniveau eines Patienten richtig einzuschätzen und ihn weder zu über- noch zu unterfordern. In die Entscheidungen zur Therapieplanung gehen damit

- Wirksamkeitsnachweise (sofern vorhanden),

- der Wille der Patienten und

- die Expertise der Sprachtherapeuten ein. Diese Faktoren bilden die Grundlage der evidenzbasierten Praxis [9], auf die im Folgenden eingegangen wird.

\section{Merke}

Die Präferenzen der Patienten, die eigene Expertise und die wissenschaftlich ermittelten Evidenzen bilden die Grundlage der evidenzbasierten Praxis. 


\section{Evidenzbasierte Praxis (EBP) in der Sprachtherapie}

Problemorientiertes Vorgehen Die EBP hat das Ziel, „in der Forschung entstandenes Wissen in die logopädische Praxis zu transportieren. Die praktisch tätige Therapeutin soll mithilfe von EBP über den aktuellen Stand der Forschung im Bilde sein, um auf dieser Basis ihre täglichen Entscheidungen in Diagnostik und Therapie zu fällen." ([10], S.7). Dafür ist es nicht notwendig, sich tagelang mit Forschungsergebnissen zu beschäftigen. Die EBP zielt vielmehr auf ein problemorientiertes Vorgehen ab: Erst dann, wenn Unsicherheiten oder Probleme auftreten, sollte die Suche nach Studienbelegen beginnen. Dabei ist es ratsam, das Problem mit einer möglichst exakten Frage zu erfassen (z.B. „Führen mundmotorische Übungen zu einer Reduktion sprechpraktisch bedingter Fehler?“). Je exakter die Frage formuliert wird, desto wahrscheinlicher ist es, aus der Literatur genau die Information zu erhalten, die benötigt wird.

Merke

Die evidenzbasierte Praxis favorisiert ein problemorientiertes Vorgehen.

Suche nach sprachtherapeutischer Evidenz Um die Suche nach sprachtherapeutisch relevanten Forschungsergebnissen zu vereinfachen, ist es empfehlenswert, zunächst in Datenbanken zu recherchieren, von denen einige in $>$ Tab. 1 aufgelistet sind. Ein Teil der Datenbanken verfügt über Suchfunktionen, die eine einfache Recherche ermöglichen. Dabei ist der Zugriff auf die Zusammenfassungen („Abtracts“) von Forschungsergebnissen in der Regel kostenlos, eine Einsicht in die dazugehörigen Volltexte kann jedoch mit Kosten verbunden sein.
FALLBEISPIEL 2: PRIORITÄT PATIENTENWILLE

In einer neurologischen Rehabilitationsklinik wird der 54-jährige Herr S. mit der Diagnose eines bilateralen Hirnstamminfarkts aufgenommen. Als Folge des Infarkts bestehen eine Stand- und Gangataxie, eine Dysarthrie sowie eine Dysphagie mit dem Aspirationsgrad IV nach Wuttge-Hannig ([8], S. 109). Aufgrund der hohen Aspirationsgefahr wird Herr S. darüber informiert, dass eine orale Ernährung nicht möglich ist. Herr S. protestiert dagegen heftig und besteht darauf, normale Nahrung zu sich zu nehmen. Er beharrt auch dann noch auf seinem Willen, nachdem ihm die (Aspirations-)Bilder der flexiblen Nasenendoskopie gezeigt und erklärt worden sind.

Da Herr S. im Alter von ca. 20 Jahren ein schweres SchädelHirn-Trauma mit nachfolgenden ausgeprägten exekutiven Störungen erlitten hat, ist bereits vor vielen Jahren eine Betreuung eingerichtet worden, die in den Händen eines Rechtsanwalts liegt. Der Rechtsanwalt wird daher darüber informiert, dass sein Mandant trotz einer hohen Aspirationsgefahr auf einer oralen Ernährung besteht. Er wird als gesetzlicher Betreuer gebeten, dem Willen des Patienten zuzustimmen. Nach Rücksprache mit dem behandelnden Sprachtherapeuten verweigert der Rechtsanwalt jedoch seine Zustimmung mit dem Hinweis auf die gesundheitlichen Risiken, die mit der oralen Ernährung verbunden sind.

Herr S. ist allerdings auch mit der Entscheidung seines Betreuers nicht einverstanden. Er sucht daher den neurologischen Chefarzt der Klinik auf, um vehement eine orale Ernährung zu fordern. Der Chefarzt entschließt sich, den Sachverhalt dem zuständigen Betreuungsgericht mit der Bitte um Klärung vorzulegen. Das Gericht kommt zu dem Schluss, dass der Wille des Patienten selbst dann zu erfüllen ist, wenn er eine gesundheitliche Gefährdung darstellt. Daraufhin erhält Herr S. Normalkost, die nach einigen Tagen zu einer Aspirationspneumonie führt. Herr S. wird daraufhin ins Akutkrankenhaus verlegt, von dem er nicht mehr zur Rehabilitation zurückkehrt.

- Tab.1 Auswahl von Datenbanken mit sprachtherapeutischen Effektivitätsnachweisen.

\begin{tabular}{|c|c|c|}
\hline Datenbank & Internetadresse & Stand \\
\hline Cochrane Collaboration & www.cochrane.org & 27.08 .2017 \\
\hline American Speech-Language-Hearing Association & www.asha.org & 27.08 .2017 \\
\hline Evidence-Based Review of Stroke Rehabiliation & www.ebrsr.com & 27.08 .2017 \\
\hline Cochrane Bibliothek & www.cochranelibrary.com & 27.08 .2017 \\
\hline Deutsches Cochrane Zentrum & www.cochrane.de & 27.08 .2017 \\
\hline Institut für Qualität und Wirtschaftlichkeit im Gesundheitswesen & www.iqwig.de & 27.08 .2017 \\
\hline $\begin{array}{l}\text { Arbeitsgemeinschaft der Wissenschaftlichen Medizinischen Fachgesellschaften } \\
\text { (Leitlinienregister) }\end{array}$ & www.awmf.org & 27.08 .2017 \\
\hline Deutscher Bundesverband für Logopädie & www.evidnessst.org & 27.08.2017 \\
\hline
\end{tabular}


- Tab.2 Sprachtherapeutisch relevante Leitlinien. Quelle: www.awmf.org

\begin{tabular}{|c|c|c|c|}
\hline Störung & Entwickelt von & Stand & AWMF-Register-Nr. \\
\hline Neurogene Dysphagie & DGN & $09 / 2011$ & $030 / 111$ \\
\hline Idiopathische Fazialisparese & DGN & $09 / 2011$ & $030 / 013$ \\
\hline Aphasie & DGN & $09 / 2012$ & $030 / 090$ \\
\hline Dysarthrie & DGN & $09 / 2012$ & $030 / 103$ \\
\hline Morbus Parkinson & DGN & $09 / 2012$ & $030 / 010$ \\
\hline Multiple Sklerose & DGN & $08 / 2014$ & $030 / 050$ \\
\hline Neurogene Sprech- und Stimmstörungen & DGPP & $08 / 2014$ & $049 / 014$ \\
\hline Pathogenese, Diagnostik und Behandlung von Redeflussstörungen & DGPP & $09 / 2016$ & $049 / 013$ \\
\hline Sprachentwicklungsstörungen & DGPP und DGKJP & $11 / 2011$ & $049 / 006$ \\
\hline Auditive Verarbeitungs- und Wahrnehmungsstörungen & DGPP & $09 / 2015$ & $049 / 012$ \\
\hline Periphere Hörstörungen im Kindesalter & DGPP & $09 / 2013$ & $049 / 013$ \\
\hline
\end{tabular}

Merke

Die Suche nach wissenschaftlichen Evidenzen sollte zunächst mit einer Literaturrecherche beginnen.

Wenn die Suche in den Datenbanken nicht ausreicht, dann bietet es sich an, medizinische Suchmaschinen zu nutzen, die es als deutsche und englische Versionen gibt. Häufig genutzte Suchmaschinen sind MEDLINE, PUBMED, CINAHL und EMBASE. In die Suchmaschinen werden sinnvolle Kombinationen von Schlüsselwörtern eingegeben, um benötigte Informationen in der nationalen und internationalen Literatur zu recherchieren. Ein Beispiel für die Suche mit Schlüsselwörtern geben ([7], S. 52). Für die Nutzung der Suchmaschinen gilt ebenfalls, dass sie zum Teil kostenpflichtig sind.

\section{Merke}

Für die Literaturrecherche können medizinische Suchmaschinen genutzt werden, die jedoch nicht kostenlos zur Verfügung stehen.

\section{Medizinische Leitlinien}

Vorteile der Leitlinien Eine weitere Quelle, die Angaben zur Effektivität von Therapiemethoden enthält, besteht aus den (medizinisch-therapeutischen) Leitlinien. Sie sind als „systematisch entwickelte Informationen oder auch Instruktionen über das diagnostische und therapeutische Vorgehen bei einem bestimmten Krankheitsbild“ definiert ([11], S. 785). Damit bieten Leitlinien Hilfen an, indem sie Empfehlungen für die Behandlung einer bestimmten (logopädischen) Störung aussprechen. Alle deutschsprachigen Leitlinien sind in dem Register der Arbeitsgemeinschaft der
Wissenschaftlichen Medizinischen Fachgesellschaften (AWMF) zu finden, auf das kostenlos zugegriffen werden kann ( $\triangleright$ Tab.1). Die Vorteile der Leitlinien sind, dass sie

- für mehr Sicherheit bei der Wahl des (richtigen) diagnostischen und therapeutischen Vorgehens sorgen,

- Angaben zur Effektivität von Therapiemethoden und zur Therapiefrequenz enthalten,

- als Referenz gegenüber Kostenträgern und verordnenden Ärzten genutzt werden können

[12]. Inzwischen gibt es eine Reihe von sprachtherapeutisch relevanten Leitlinien, die in $>$ Tab. 2 aufgelistet sind.

\section{Merke}

Leitlinien enthalten in der Regel das aktuell beste Wissen über die Behandlung einer bestimmten Störung.

Umsetzung der Leitlinien Die Vorteile der EBP für klinische Entscheidungen ist zwar offensichtlich, dennoch „ist [es] (...) nicht immer einfach, Studienergebnisse (...) aus der klinischen Forschung in die (sprach)therapeutische Praxis zu übertragen“ ([12], S. 34). Ein Grund dafür liegt darin, dass die klinische Forschung in der Regel unter idealtypischen Bedingungen stattfindet, die dem therapeutischen Alltag mit all seinen nicht kontrollierbaren Einflüssen nur unvollständig entspricht. Daher kann der Aufwand zur Umsetzung eines Forschungsergebnisses in der Praxis so hoch sein, dass er sich nicht realisieren lässt (Ineffizienz). Ein weiterer Grund ist, dass es das Ziel der Forschung ist, zu allgemeingültigen Aussagen zu kommen (hohe interne Vali- 
dität). Der Einzelfall interessiert dabei wenig (geringe externe Validität). In der Praxis sind jedoch immer Überlegungen anzustellen, die sich auf die Situation eines individuellen Patienten beziehen. Zwischen der individuellen Situation und einem bestimmten Forschungsergebnis muss allerdings keine Übereinstimmung vorliegen. Damit hat das (Forschungs)-Ideal der hohen internen Validität den Nachteil, dass es zu einer geringen externen Validität führt. Ein letzter Grund besteht darin, dass es Widersprüche zwischen aktuellen Forschungsergebnissen und den gesetzlichen Rahmenbedingungen geben kann, unter denen Sprachtherapie stattfindet [13]. Dies illustriert das Fallbeispiel 3.

Offensichtlich dauert es, bis Forschungsergebnisse in die gesetzlichen Rahmenbedingungen eingehen. Dabei handelt es sich jedoch nicht nur um ein Problem in Deutschland, sondern auch in den anglo-amerikanischen Ländern. Eine Übersicht darüber, welche Rahmenbedingungen in der Sprachtherapie für eine effektive Therapie benötigt werden, gibt [17].

Merke

Es mag aus unterschiedlichen Gründen länger dauern, bis die Inhalte einer Leitlinie in die praktische Versorgung eingehen.

Trotz der Hindernisse, die sich der Umsetzung der EBP in den Weg stellen, ist sie ein unverzichtbarer Bestandteil klinischer Entscheidungen. Denn die EBP trägt dazu bei, dass Entscheidungen nicht auf bloßen Vermutungen beruhen, sondern auf Fakten, die wissenschaftlich abgesichert sind. Sie hat zusätzlich den Vorteil, das Wissen der Therapeuten kontinuierlich zu erweitern und damit zu einer qualitativ hochwertigen Sprachtherapie zu führen.

\section{Qualitätssicherung}

Verpflichtende Qualitätssicherung Mit den wissenschaftlich ermittelten Evidenzen werden den (Sprach)Therapeuten starke Argumente in die Hände gegeben, um fundierte Entscheidungen treffen zu können. Die EBP stellt außerdem sicher, dass die Anforderungen der Qualitätssicherung umgesetzt werden, die für alle verpflichtend sind, die medizinisch-therapeutische Leistungen erbringen. So erwartet der Gesetzgeber, dass (therapeutische) Leistungen „dem jeweiligen Stand der wissenschaftlichen Erkenntnisse entsprechen und in der fachlich gebotenen Qualität erbracht werden“ ( $135 a$ Abs. 1 Sozialgesetzbuch V). Die Qualitätssicherung ist jedoch nicht nur gesetzlich vorgeschrieben, sondern stellt auch eine freiwillige Selbstverpflichtung der Logopäden dar. Im Leitbild Logopädie heißt es dazu, dass „Logopädinnen und Logopäden [...] den Stand ihres Fachwissens und die Ergebnisse ihrer beruflichen Tätigkeit kontinuierlich reflektieren.“
FALLBEISPIEL 3: UNSTIMMIGKEIT ZWISCHEN FORSCHUNGSERGEBNISSEN UND RAHMENBEDINGUNGEN Um die Frequenz der Aphasietherapie zu erhöhen, können zusätzlich zur klassischen Einzeltherapie computerbasierte Therapien eingesetzt werden, für die inzwischen einige Effektivitätsnachweise existieren [14]. Die Computertherapie lässt sich jedoch in der stationären neurologischen Rehabilitation nicht zulasten der Deutschen Rentenversicherung abrechnen, da sie in der derzeit gültigen Klassifikation therapeutischer Leistungen (KTL-Katalog) nicht enthalten ist [15]. Ebenso wenig können Eigenübungsaufgaben oder die Supervision von Laientherapien abgerechnet werden, obwohl eine supervidierte Laientherapie durchaus effektiv ist [16].

[18]. Die Übernahme der EBP in die tägliche Praxis gilt damit für alle Sprachtherapeuten unabhängig davon, ob sie in stationären, teilstationären oder ambulanten Einrichtungen arbeiten.

Merke

Für stationäre und teilstationäre Einrichtungen ist eine Beteiligung an der Qualitätssicherung gesetzlich vorgeschrieben.

\section{Wirksamkeitsnachweise für die Aphasietherapie}

Obwohl das Wissen über die Effektivität logopädischer Therapiemethoden (noch) begrenzt ist [7], gibt es für die Rehabilitation der Aphasien einige therapeutische Prinzipien, für die Wirksamkeitsnachweise vorliegen $[12,19]$. Zu ihnen gehören:

- Ein früher Therapiebeginn: Die sprachlichen Fortschritte sind am größten, wenn die Therapie unmittelbar nach Krankheitsbeginn einsetzt.

- Eine hohe Therapiefrequenz: Aphasietherapie ist dann effektiv, wenn sie mit mindestens 300 Min. Therapie pro Woche für einen Zeitraum von 9-12 Wochen durchgeführt wird.

- Repetition: Die wiederholte Darbietung identischer Therapieinhalte ist effektiver als ein ständiger Wechsel.

- „Shaping“: Im Therapieverlauf sind die Anforderungen an den Patienten kontinuierlich zu erhöhen, indem therapeutische Hilfen schrittweise reduziert oder Aufgaben mit zunehmendem Schwierigkeitsgrad durchgeführt werden.

- „Design of learning situation“: Die Therapieinhalte sind konkret, nicht jedoch abstrakt zu präsentieren. Denn abstrakte Aufgaben (z. B. die Suche nach Reimwörtern), die sich nur mit einer (kognitiven) Distanz zur jeweiligen Situationen lösen lassen, stellen für Patienten mit einer Aphasie eine sehr viel 
größere Herausforderung dar als konkrete Aufgaben (z. B. die Vervollständigung automatisierter sprachlicher Reihen), die unmittelbar, situativ und intuitiv bewältigt werden können.

Es wird derzeit geprüft, ob diese Prinzipien auch außerhalb der Aphasietherapie wirksam sind. Die Ergebnisse einiger Untersuchungen deuten dabei darauf hin, dass v.a. die hohe Therapiefrequenz ein Faktor zu sein scheint, der auch für die Therapie von Sprechapraxien, Sprachentwicklungsstörungen und Stimmstörungen von entscheidender Bedeutung ist (Überblick in [12]). Der Frequenz sollte daher bei der Planung sprachtherapeutischer Interventionen eine hohe Priorität zukommen.

\section{Merke}

Die Therapiefrequenz spielt für den Behandlungserfolg eine außerordentlich wichtige Rolle.

\section{Bedeutung evidenzbasierter Entscheidungen}

Mit der Zunahme an wissenschaftlichen Evidenzen werden die sprachtherapeutischen Denk- und Entscheidungsprozesse zukünftig immer sicherer werden. Dies hilft zum einen, wenn im Konfliktfall Entscheidungen zu legitimieren sind. Zum anderen bilden die Evidenzen das sprachtherapeutische Leistungs- und Wirkungsspektrum ab. Sie tragen damit zum Renommee der Sprachtherapie bei, indem der Glaube an die Wirksamkeit sprachtherapeutischer Interventionen durch wissenschaftlich ermittelte Effektivitätsnachweise ersetzt wird.

Merke

Evidenzbasierte Entscheidungen erhöhen den Behandlungserfolg und verbessern dadurch das Renommee der Sprachtherapie.

\section{FAZIT FÜR DIE PRAXIS}

Was war bekannt?

- Sprachtherapeuten haben das Ziel, effiziente und effektive Therapien durchzuführen. Dazu greifen sie auf ihr Wissen zurück, das sie sich in der Berufsausbildung und in Fort- und Weiterbildungen angeeignet haben.

- Bei der Therapieplanung berücksichtigen sie die Interessen der Patienten.

- Eine Therapie ist dann erfolgreich, wenn gezeigt werden kann, dass die Therapieziele erreicht worden sind.

Was war neu?

- Zur Planung wirksamer Therapien stehen v. a. wissenschaftlich ermittelte Evidenzen und Leitlinien zur Verfügung.

- Therapeutische Entscheidungen, die auf wissenschaftlichen Evidenzen beruhen, schaffen Sicherheit und minimieren das Fehlerrisiko.

- Die evidenzbasierte Praxis ist ein probates Mittel, um auf therapeutische Fragen eine Antwort zu erhalten.

\section{Schlüsselwörter}

Effektivität, Evidenz, Leitlinien, Qualitätssicherung

Interessenkonflikt

Der Autor gibt an, dass kein Interessenkonflikt besteht.

\section{Autorinnen/Autoren}

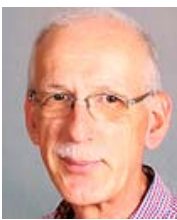

\section{Holger Grötzbach}

M.A., Leiter der Sprachtherapie der Asklepios Klinik Schaufling. Arbeitsschwerpunkte: Diagnose und Therapie der Aphasien, evidenzbasierte Sprachtherapie, Umsetzung der ICF

Institut

Abteilung Neurologie, Asklepios Klinik Schaufling, Schaufling 


\section{Korrespondenzadresse}

Holger Grötzbach, M. A.

Abteilung Sprachtherapie

Asklepios Klinik Schaufling

Hausstein 2

94571 Schaufling

E-Mail: h.groetzbach@asklepios.com

\section{Literatur}

[1] Beushausen U. Von der Anfängerin zur Expertin. In: Beushausen $U$, (Hrsg.) Therapeutische Entscheidungsfindung in der Sprachtherapie. München: Urban \& Fischer; 2009: 2938

[2] Grötzbach H. Zielsetzung in der Aphasietherapie. Forum Logopädie 2004; 18: 2 -6

[3] Grötzbach H. Evaluationsstudien bei der Therapie von Aphasien. In: Grohnfeldt M ; Hrsg. Kompendium der akademischen Sprachtherapie und Logopädie. Band 4: Aphasien, Dysarthrien, Sprechapraxie, Dysphagien, Dysphonien Stuttgart: Kohlhammer; im Druck

[4] Bauer A, de Langen-Müller U, Glindemann R et al. Qualitätskriterien und Standards für die Therapie von Patienten mit erworbenen neurogenen Störungen der Sprache (Aphasie) und des Sprechens (Dysarthrie). Aktuelle Neurologie 2002; 29: $63-75$

[5] Baumgärtner A. Intensität in der Aphasietherapie. In: Grötzbach $\mathrm{H}$; Hrsg. Intensität in der Sprachtherapie. Idstein: Schulz-Kirchner; 2017: 41-68

[6] Breitenstein C, Grewe T, Flöel A et al. Intensive speech and language therapy in patients with chronic aphasia after stroke: a randomised, open-label, blinded-endpoint, controlled trial in a health-care setting. 2017: http://dx.doi. org/10.1016/50140-6736(17)30067-3

[7] Beushausen U, Grötzbach H. Evidenzbasierte Sprachtherapie. München: Elsevier; 2011

[8] Wuttge-Hannig A, Hannig C. Radiologische Funktionsdiagnostik von Schluckstörungen bei neurologischen Krankheitsbildern und bei therapierten onkologischen Kopf-HalsErkrankungen. In: Bartolome H, Schröter-Morasch H, Hrsg. Schluckstörungen. Diagnostik und Rehabilitation. München: Urban \& Fischer; 2006: 99 - 154

[9] Dollaghan C. The handbook of evidence-based practice in communication disorders. Baltimore: Paul H. Books; 2007

[10] Beushausen U. Evidenz-basierte Praxis in der Logopädie Mythos und Realität. Forum Logopädie 2005; 19 : 6-11
[11] Lüthi H, Blanco ], Mäder M. Dokumentation, Messung und Qualitätsmanagement. In: Frommelt P, Lösslein H, Hrsg. Neuro-Rehabilitation. Berlin: Springer; 2010: 771- 788

[12] Grötzbach H, Hrsg. Intensität in der Sprachtherapie. Idstein: Schulz-Kirchner; 2017

[13] Beushausen U, Grötzbach H. More is better! Intensität in der Sprachtherapie Forum Logopädie 2017; 31: 28 - 35

[14] Nobis-Bosch R, Springer L, Radermacher I et al. Supervised home training of dialogue skills in chronic aphasia: a randomized parallel group study. Journal of Speech, Language, and Hearing Research 2011; 54: $1118-1136$

[15] Deutsche Rentenversicherung Bund. Klassifikation therapeutischer Leistungen in der medizinischen Rehabilitation. Berlin: Buck; 2015

[16] de Langen-Müller U. Angehörigenarbeit bei Aphasie: Brauchen oder können Laien Therapie? Formen und Wirksamkeit der indirekten Aphasietherapie Stimme - Sprache - Gehör 2015; 39: $123-128$

[17] Winterholler C. Intensität in der Sprachtherapie im Spannungsfeld von Forschung-Gesetzgebung-Allokation. In: Grötzbach H, Hrsg. Therapie Intensität in der Sprachtherapie/Logopädie. Idstein: Schulz-Kirchner; 2017: 235-250

[18] Leitbild Logopädin/Logopäde. Forum Logopädie 2005; 19 : 37

[19] Grötzbach H, Spitzer L. Was bedeuten aphasische Symptome? Die Position von Goldstein und Gelb Neurologie \& Rehabilitation 2015; 21: 329-335

Bibliografie

DOI https://doi.org/10.1055/a-0625-5882

Sprache · Stimme · Gehör 2018; 42: 133-139 (c) Georg Thieme Verlag KG Stuttgart · New York ISSN 0342-0477 